\title{
DRESS syndrome (drug rash with eosinophilia and systemic symptoms): sometimes, not all sigms are there
}

\author{
Fábio Murteira, Pedro Oliveira, João Miranda, Albina Moreira, Margarida Correia \\ Internal Medicine Department, Centro Hospitalar Vila Nova de Gaia/Espinho, Porto, Portugal
}

\begin{abstract}
DRESS syndrome is a rare and potentially fatal multisystemic reaction that occurs two to six weeks after exposure to certain drugs. It is characterized by fever, eosinophilia, and skin rash. Case of a 37-year-old man, black, with gout (started allopurinol 4 weeks before). He arrived at the Emergency Room complaining of headache, fever, abdominal pain, and scattered maculopapular skin lesions. The conducted study showed elevated transaminases and C-reactive protein, hepatomegaly, and peri-hepatic adenomegalies. Allopurinol was stopped, but throughout hospitalization, liver, renal and neurological dysfunctions (with meningoencephalitis) worsened; it also appeared eosinophilia. A skin biopsy was performed, the possibility of DRESS syndrome was assumed, and methylprednisolone $2 \mathrm{mg} / \mathrm{kg}$ /day was started, with progressive improvement. The skin biopsy was compatible with DRESS. The described case reveals some peculiarities. Among the most relevant arethe late onset of eosinophilia and the difficulty in evaluating the rash due to the patient's skin tone and neurological manifestations (rare).
\end{abstract}

\section{Introduction}

DRESS (drug rash with eosinophilia and systemic symptoms) syndrome is a rare and potentially fatal systemic reaction that occurs after exposure to some drugs, with the most frequently involved agents are

Correspondence: Fábio José da Silva Murteira, Internal Medicine Department, Centro Hospitalar Vila Nova de Gaia/Espinho, Porto, Estrada Nacional 327, 399 B, Santa Maria da Feira, 4520-706, Portugal.

Tel.: +351.912939969. E-mail: fabio.murteira@gmail.com

Key words: DRESS syndrome; eosinophilia; allopurinol; rash.

Contributions: FM, contributed to the clinical management of the patients, wrote the first draft, and managed all the submission processes. PO and JM, contributed to the clinical management of the patient and wrote the first draft. AM and MC, contributed to the clinical management of the patient and revised the manuscript.

Conflict of interests: the authors declare no conflict of interests.

Ethical approval and consent: the authors have no ethical conflicts to disclose. Informed consent was obtained from the patient.

Received for publication: 7 February 2021.

Accepted for publication: 7 February 2021.

This work is licensed under a Creative Commons Attribution NonCommercial 4.0 License (CC BY-NC 4.0).

${ }^{\circ}$ Copyright: the Author(s), 2021

Licensee PAGEPress, Italy

Italian Journal of Medicine 2021; 15:175-178

doi:10.4081/itjm.2021.1475 some anticonvulsants allopurinol. ${ }^{1,2}$ It is typically characterized by a long period between exposure to the drug and the onset of symptoms (two to six weeks), ${ }^{3}$ with one case in every 1000 to 10,000 exposures. ${ }^{1}$ Fever, eosinophilia, and skin rash are often the defining signs of the disease, being present in most cases. All organ systems can be involved (liver, kidney, and lung involvement are the most frequent), and there is significant variability of manifestations between patients. ${ }^{3,4}$ Since the evolution can be fulminating, its rapid clinical diagnosis is important so the treatment can be started as soon as possible. ${ }^{5}$ The presented case stands out for the rarity of involvement of some organs and the delay in disclosing defining characteristics (such as eosinophilia).

\section{Case Report}

We present the case of a 37-year-old man, black, born in Angola but living in Portugal for 23 years (no travels recently), with pathological history of hyperuricemia and gout. Four weeks before, he started allopurinol $300 \mathrm{mg} /$ day.

He arrived at the Emergency Room (ER) after complaining over the two days before, of holocranial headache, fever, anorexia, myalgia, erythematous, and pruritic maculopapular lesions scattered throughout the body, and belt-like pain in the upper quadrants of the abdomen.

Upon admission to the ER, he presented feverish $\left(39.3^{\circ} \mathrm{C}\right)$, with generalized abdominal pain and cutaneous lesions observed throughout the upper limbs and trunk, with confluence areas, sparing palms, and soles. The conducted study showed elevated transaminases 
(two times normal) and C-reactive protein; no other changes in the remaining analytical study. Abdominal and pelvic computed tomography (CT) demonstrated: hepatomegaly, periportal edema, and adenomegalies in the hepatic hilum. He was admitted for the study of the clinical condition, and allopurinol was stopped since this entrance.

On the first days, the cutaneous lesions persisted, being no longer erythematous (Figure 1), and he presented daily fever spikes and abdominal pain that was difficult to control.

Various blood cultures sets were performed without any isolation. During hospitalization, several infectious causes were excluded (Table 1), and only the serology of human herpesvirus 6 (HHV6) was compatible with reactivation.

On the $3^{\text {rd }}$ day of hospitalization, anemia appeared (hemoglobin $10.4 \mathrm{~g} / \mathrm{dL}$, normocytic, normochromic, without nutritional deficits, ferritin of $2000 \mathrm{ng} / \mathrm{mL}$ ) and leukocytosis $(13,540 / \mathrm{UL})$ with eosinophilia (1100/UL). In addition, due to the worsening of transaminases (five times normal), the appearance of hyperbilirubinemia $(3.9 \mathrm{mg} / \mathrm{dL}$, at the expense of direct), and continuous elevation of inflammatory parameters, he repeated abdominal-pelvic CT, which showed aggravated hepatomegaly $(21 \mathrm{~cm})$ and infarction in the right kidney subcapsular area. Antiphospholipid antibodies were performed, showing only positive lupus anticoagulant;

Table 1. Other infectious diseases excluded.

\begin{tabular}{ll}
\hline Other infectious diseases excluded & Parvovirus \\
\hline Human immunodeficiency virus 1 and 2 & Group A Streptococcus \\
\hline Eytomegalovirus & Toxoplasma gondii \\
\hline Herpes simplex virus 1 and 2 & Rickettsia conorii \\
\hline Hepatitis A virus & Leptospira \\
\hline Hepatitis B virus & Schistosoma \\
\hline Hepatitis C virus & Strongyloides stercoralis \\
\hline Hepatitis E virus & Toxocara \\
\hline Human T-cell lymphotropic viruses 1 and 2 & Hepatic fasciola \\
\hline Bacterial endocarditis (with septic embolization) & Parasites (blood and stool)
\end{tabular}
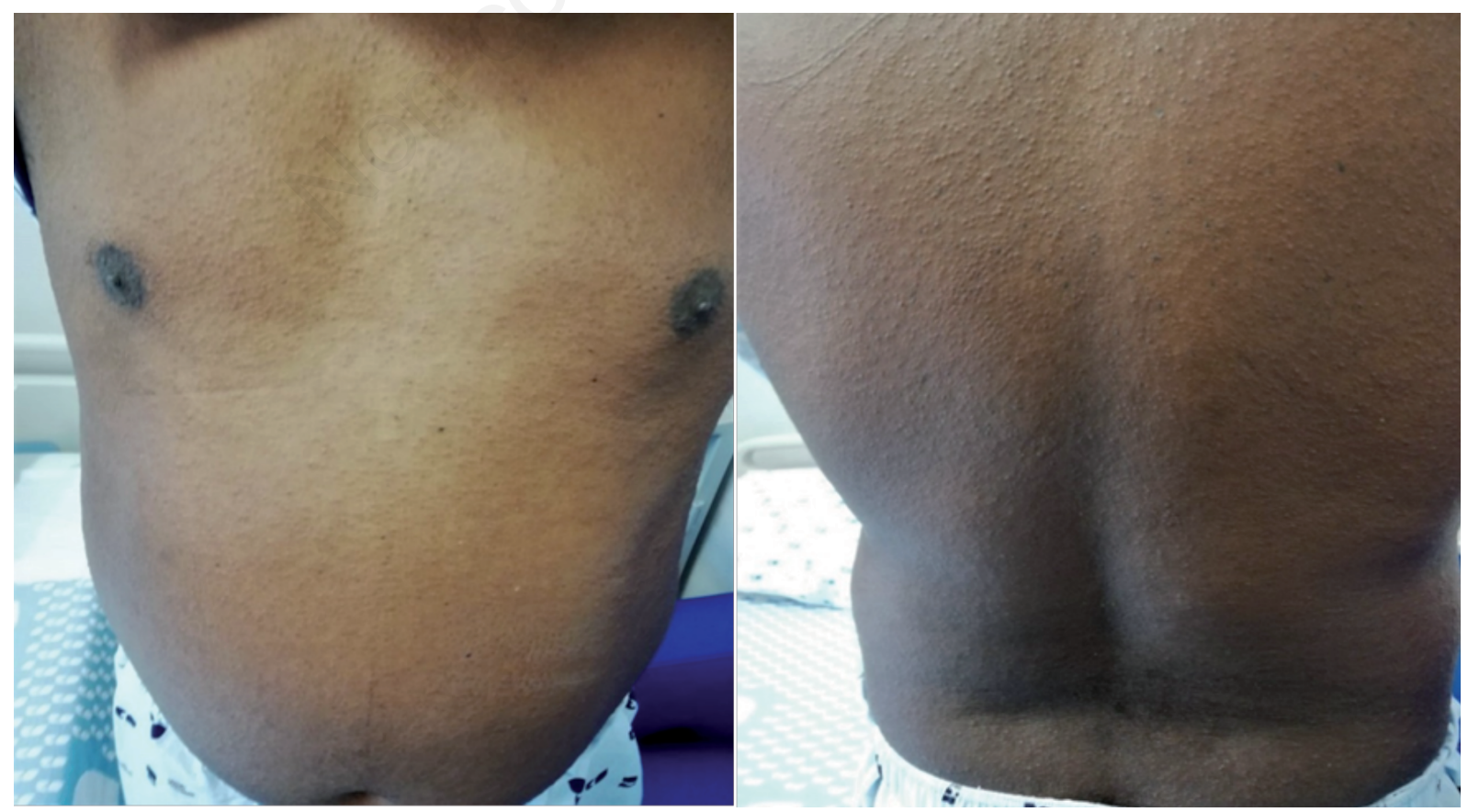

Figure 1. Maculopapular lesions on admission to hospital. 
the remaining autoimmune study was normal. He also did not show any signs of intravascular hemolysis, significant changes in the coagulation study, or fibrinogen consumption.

On the $5^{\text {th }}$ day of hospitalization, he developed oliguria, acute kidney injury AKIN 3 , urinary sediment with proteinuria $(200-650 \mathrm{mg} / \mathrm{dL})$, subtle leukocyturia, and erythrocyturia as well as generalized edema. As the oliguria persisted and the renal function worsened, he started hemodialysis. Also, noteworthy were the worsening eosinophilia (2730/UL) and the evolution of some maculopapular lesions to pustular lesions (Figure 2). Therefore, a skin biopsy was performed.

On the $8^{\text {th }}$ day of hospitalization, he had an episode of acute confusional syndrome, without hyperammonemia, asterixis, or focal deficits, but visual and auditory hallucinations. Brain CT scan did not show any change, but cerebrospinal fluid (CSF) cytochemical analysis (with normal glycorrhachia, elevated proteinorrhachia, and pleocytosis with a predominance of monomorphonuclear cells) was compatible with meningoencephalitis, despite negative bacteriological and virological tests (Enterovirus, Herpes simplex 1 and 2 and Varicella Zoster).

Despite the difficulty in evaluating skin lesions and a somewhat atypical evolution with some organic conditions that did not allow to rule out other etiologies completely, given the recent onset of allopurinol, the possibility of DRESS syndrome was assumed (European Registry of Severe Cutaneous Adverse Reactions [RegiSCAR] score 9). This hypothesis led to the empirical initiation of high-dose methylprednisolone (2 $\mathrm{mg} / \mathrm{kg} /$ day). After the start of the treatment, he progressively improved with resolution of fever, neurological normalization, improvement of liver parameters, and renal function; thus, hemodialysis was suspended. The result of the skin biopsy was later available, which was compatible with DRESS syndrome.

He maintained a progressive improvement in renal function and skin lesions until discharge, even when weaning corticosteroid therapy. After 2 weeks, he was re-evaluated in a consultation: he had no complaints, revealed a total disappearance of the cutaneous lesions and a marked improvement in creatinine $(1.2 \mathrm{mg} / \mathrm{dL})$. Nevertheless, he maintained weaning of corticosteroid therapy and regular follow-up.

\section{Discussion}

The DRESS syndrome is part of the Severe Cutaneous Adverse Reactions (SCAR) group, including Stevens-Johnson Syndrome, Toxic Epidermal Necrolysis, and Acute Exanthematous Generalized Pustulosis, being distinguished from these by their specific manifestations. ${ }^{6}$ It presents with dispersed morbilliform rash, eosinophilia, systemic symptoms (mainly fever), facial edema, lymphadenopathies, and multiorgan involvement. ${ }^{1}$ The presented case shows all these characteristics. Therefore, according to the RegiSCAR score, a value $\geq 6$ makes the definitive diagnosis of DRESS. ${ }^{7}$ In this case, we have a score of 9, reflecting diagnostic certainty.

Although eosinophilia appeared only during hospitalization (typically found at presentation) and after discontinuation of the drug, all organic conditions met the expected latency time, albeit asynchronously.

Even under appropriate treatment, DRESS has a mortality rate up to $10 \%{ }^{1}$ higher in cases related to allopurinol (12-27\%) which tend to be more severe. ${ }^{2}$ Liver dysfunction is the most frequent and the leading cause of mortality. ${ }^{1}$

Because the clinical manifestations mimic other eti-

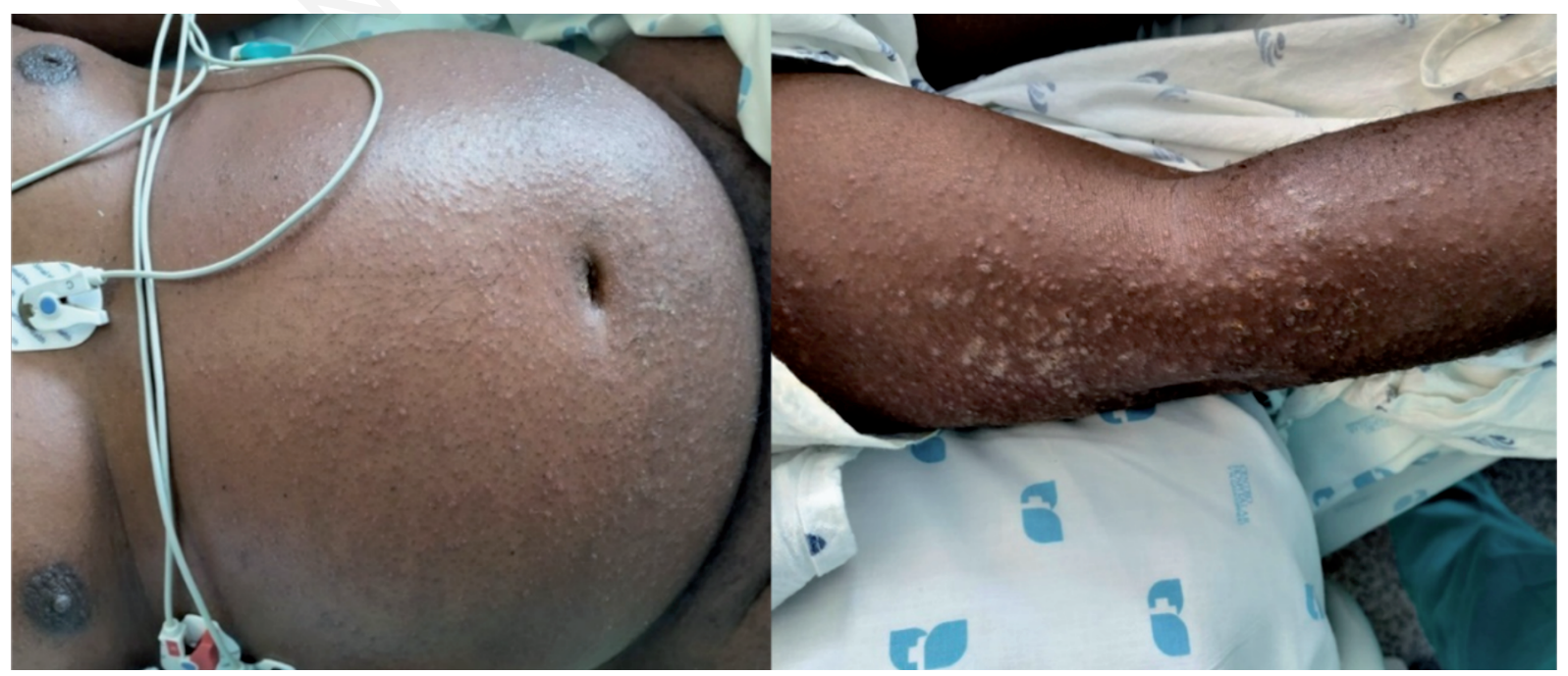

Figure 2. Progression from maculopapular lesions to pustular lesions (abdomen and arm). 
ologies, the differential diagnosis is complex and can delay treatment. The absence of eosinophilia and facial edema in the first days, the absence of palpable adenomegalies (a rarer sign with allopurinol ${ }^{2}$ ), as well as the finding of renal infarction, may have helped to delay our diagnosis. The patient's skin tone also makes the rash more tenuous, which may have reduced the diagnostic accuracy. In a minority of cases, maculopapular lesions develop into pustules, ${ }^{8}$ as occurred in this case.

Despite being under proper treatment when the result was available, the skin biopsy (although unspecific) ended up helping to establish the diagnosis. HHV-6 reactivation, which is frequent in this syndrome ${ }^{1}$, has also been documented. It would have been interesting to assess the carrier status of the human leukocyte antigen (HLA) B58*01 allele, associated with skin reactions induced by allopurinol. ${ }^{9}$

In this case, the renal infarction is probably directly related to the DRESS syndrome. Thrombotic events are described but are rarely associated with DRESS,${ }^{10}$ which demonstrates the uniqueness of this case. The positivity of the lupus anticoagulant may be due to the pro-inflammatory state and not a primary alteration, being planned to repeat it after the complete disease resolution.

Another peculiarity was the rarely described neurological manifestation, ${ }^{11,12}$ which may have several causes: metabolic encephalopathy due to concomitant dysfunctions, focal epilepsy due to possible phenomena of microvasculitis ${ }^{13}$ with associated hallucinations, and meningoencephalitis (compatible CSF analysis) caused by reactivation of HHV-6. ${ }^{12}$ It is impossible to prove these hypotheses because the study has not been performed (such as electroencephalogram, angiographic study, or HHV-6 research in CSF) due to the rapid improvement of dysfunctions after the start of corticosteroid therapy.

In addition to the interruption of the causative agent (since the beginning of hospitalization in our case), the most used treatment is a high-dose systemic corticosteroid $(0.5-2 \mathrm{mg} / \mathrm{kg} /$ day of prednisolone or equivalent). ${ }^{6,7}$ Despite the absence of studies that prove its efficacy, in this case, there was an apparent response to corticosteroid therapy without the need for association with other immunosuppressants. Maintenance of a high dose until clinical improvement is indicated, with subsequent slow weaning (2-3 months) due to the apparent relationship between rapid weaning and the risk of relapse..$^{14}$ It is important to maintain posterior follow-up due to a consistent correlation with the appearance in the medium term of autoimmune diseases. ${ }^{15}$

\section{Conclusions}

Despite its known clinical characteristics and welldefined diagnostic criteria, DRESS syndrome remains a medical challenge due to its different forms of pres- entation, evolution, and mimicry with other pathologies. In case of suspicion, the drug should be stopped immediately, corticosteroid therapy initiated, and tight monitoring performed. Allopurinol is one of the most commonly associated drugs; thus its use should be limited to cases of real need. It may be essential to research the HLA allele (B58*01) to which hypersensitivity reactions to this drug are regularly associated. Controlled and randomized studies are still needed to evaluate the effectiveness and define the best therapeutic regimen to be used.

\section{References}

1. Isaacs M, Cardones R, Rahnama-Moghadam S. Dress syndrome: clinical myths and pearls. Cutis 2018;102:322-6.

2. Adwan MH. Drug reaction with eosinophilia and systemic symptoms (DRESS) syndrome and the rheumatologist. Curr Reumathol Rep 2017;19:1-9.

3. Kardaun SH, Sekula P, Valeyrie-Allanore L, et al. Drug reaction with eosinophilia and systemic symptoms (DRESS): an original multisystem adverse drug reaction. Results from the prospective RegiSCAR study. $\mathrm{Br}$ J Dermatol 2013;169:1071-80.

4. Behera S, Das S, Xavier A, Selvarajan S. DRESS syndrome: a detailed insight. Hospital Pract 2018;46:152-62.

5. Husain Z, Reddy BY, Schwartz RA. DRESS syndrome: Part II. Management and therapeutics. J Am Acad Dermatol 2013;68:709.e1-9.

6. Cacoub P, Musette P, Descamps V, et al. The DRESS syndrome: a literature review. Am J Med 2011;124:588-97.

7. Kardaun SH, Sidoroff A, Valeyrie-Allanore L, et al. Variability in the clinical pattern of cutaneous side-effect of drugs with systemic symptoms: Does DRESS syndrome really exist? Br J Dermatol 2007;156:609-11.

8. Corneli HM. DRESS syndrome: drug reaction with eosinophilia and systemics symptoms. Pediatr Emerg Care 2017;33:499-504.

9. Gonçalo M, Coutinho I, Teixeira V, et al. HLA-B*58:01 is a risk factor for allopurinol-induced DRESS and Stevens-Johnson syndrome/toxic epidermal necrolysis in a Portuguese population. Br J Dermatol 2013;169: 660-5.

10. LaHood N, Sokol K. DRESS syndrome associated with splenic thrombosis. Ann Allergy Asthma Immunol 2017;119:463-4.

11. Husain Z, Reddy BY, Schwartz RA. DRESS syndrome: Part I. Clinical perspectives. J Am Acad Dermatol 2013;68:693.e1-14.

12. Masaki T, Fukunaga A, Tohyama M, et al. Human herpes virus 6 encephalitis in allopurinol-induced hypersensitivity syndrome. Acta Derm Venereol 2003; 83:128-31.

13. Gaha M, Landry D, Bélair M, et al. DRESS syndrome: cerebral vasculitic-like presentation. Neuroradiology 2015;57:1015-21.

14. Tas S, Simonart T. Management of drug rash with eosinophilia and systemic symptoms (DRESS syndrome): an update. Dermatology 2003;206:353-6.

15. Chen YC, Chang CY, Cho YT, et al. Long-term sequelae of drug reaction with eosinophilia and systemic symptoms: A retrospective cohort study from Taiwan. J Am Acad Dermatol 2013;68:459-65. 